\title{
The Return-to-Sport Clearance Continuum Is a Novel Approach Toward Return to Sport and Performance for the Professional Athlete
}

\author{
Pete Draovitch, D.P.T., S.C.S., Snehal Patel, M.P.T., S.C.S., William Marrone, D.P.T., S.C.S., \\ M. Jake Grundstein, D.P.T, M.B.A., Reg Grant, C.A.T(C)., R.S.C.C.*E., \\ Adam Virgile, M.S., C.S.C.S., Tom Myslinski, Asheesh Bedi, M.D., James P. Bradley, M.D., \\ Riley J. Williams III, M.D., Bryan Kelly, M.D., and Kristofer Jones, M.D.
}

\begin{abstract}
The concept of returning to sport for a professional athlete is still under debate for the professional athlete in today's sports environment. It is critical for the professional athlete to be able to return to sport at a highly competitive level but also to return in a safe and timely measure. With no "gold standard" of sport testing, it is difficult to determine what the right progression or testing regimen should be. The Return to Sport Clearance Continuum does not look at one moment in time, but looks throughout the continuum of healing to determine readiness for sport. The purpose of this article is to explore the concept of RTS being part of an evolving continuum rather than the traditional notion that RTS is a single decision made at a discrete point in time. The principles of progressive but regular testing procedures including qualitative and quantitative movement are presented to help the professional athlete return to sport at their maximal performance level. Level of Evidence: V, expert opinion.
\end{abstract}

$\mathbf{O}$ ne of the hardest things to do as a physician or clinician is to determine when an athlete has been cleared to return to sport (RTS). There are many factors that contribute to an athlete's readiness to RTS,

Sports Rehabilitation and Performance, Jacksonville Jaguars, Jacksonville, Florida (P.D.); Sports Rehabilitation and Performance Department (S.P., W.M., M.J.G.) and Sports Medicine and Hip Preservation Service (B.K.), Hospital for Special Surgery, New York, New York; Human Performance Division, Holy Name Medical Center, Teaneck, New Jersey (R.G.); College of Nursing and Health Sciences, University of Vermont, Burlington, Vermont (A.V.); Ann Arbor, Michigan (T.M.); Sports Medicine and Shoulder Surgery, University of Michigan, Ann Arbor, Michigan (A.B.); Orthopedics, University of Pittsburgh (J.P.B.) and Head Team Physician, Pittsburgh Steelers (J.P.B.), Pittsburgh, Pennsylvania; Hospital for Special Surgery-Weill Cornell Medical College, New York, New York (R.J.W.); and Department of Orthopedic Surgery, Division of Sports Medicine, David Geffen School of Medicine, University of California Los Angeles, Los Angeles, California (K.J.), U.S.A.

The authors report that they have no conflicts of interest in the authorship and publication of this article. Full ICMJE author disclosure forms are available for this article online, as supplementary material.

Received August 23, 2021; accepted October 29, 2021.

Address correspondence to Snehal Patel, P.T., M.P.T., S.C.S., Sports Medicine Institute, Hospital for Special Surgery, 610 West 58th St., New York, NY 10019.E-mail: patelsn@hss.edu

Published by Elsevier on behalf of the Arthroscopy Association of North America. This is an open access article under the CC BY-NC-ND license (http://creativecommons.org/licenses/by-nc-nd/4.0/).

2666-061X/211256

https://doi.org/10.1016/j.asmr.2021.10.026 including muscular strength, cardiovascular fitness, balance, and psychological readiness. Monitoring progress in these domains allows practitioners to make more informed training decisions and increase the probability that the athlete is able to RTS at a high level of performance. Technological advancements have made it possible for medical, rehabilitation, and performance communities to easily collect information that can be used to improve athlete care and development as they prepare for RTS. However, interpreting the value and application of the collected information can remain an ongoing struggle.

The purpose of this article is to explore the concept of RTS being part of an evolving continuum rather than the traditional notion that RTS is a single decision made at a discrete point in time. Researchers and statisticians are critical to informing RTS through experimental study design, execution, and analysis, allowing for important risk factors of successful RTS to be identified. However, clinical competency is where "the rubber meets the road": clinicians must collect, manage, analyze, and interpret information in real time to ensure appropriate athlete management, activity modification, exercise form, and progression. These are essential as the first line of defense for RTS. Using an interdisciplinary approach, medical experts, and other 
stakeholders in the athlete's development have the capacity to integrate technology within rehabilitation and performance to monitor and address clinically relevant obstacles. Given that internal and external factors influence an athlete's response to training and RTS readiness from day-to-day progression or regression, they must be identified to ensure both safety and optimization of performance potential. Some of the current literature in looking at "Return to Sport" uses semantics such as Chaos Continuum, whereas an abundance of the anterior cruciate ligament (ACL) injury literature uses time- and impairment-based measures as criteria for returning. ${ }^{1-3}$ While RTS decisions being made without technology may have needed to rely on such means, today's technologythriving world allows for clinicians to merge that clinical judgment with corresponding numerical data to make informed decisions. Failure to integrate sound, experienced clinical judgment, alongside technology and an ebb and flow continuum philosophy may have us asking the same questions about returning from injury for years to come.

\section{Fundamental Movement}

The fundamental goal for allowing anyone to advance through the RTS process is that they be able to move in a pain-free, uncompensated fashion. For successful RTS to occur, the athlete must be able to execute movement patterns present in his or her sport without pain and without performance-limiting compensations. Sequencing and synchronizing the movements of body segments to execute physical tasks allows for efficient transfer of forces throughout the body, resulting in powerful movements required for successful sport performance. This eloquent synchrony of precise movements has been termed "kinetic linking" and is essential for high-quality execution of sporting skills, such as throwing, hitting, kicking, jumping, and running. Kibler reports that 3 kinematic chain elements must exist for kinetic linking to occur. ${ }^{4}$ These elements include the following: (1) functionally intact anatomy; (2) appropriate physiological muscle activation; and (3) appropriate biomechanical forces and motions that result in a desired athletic function.

The inability to exhibit these qualities can stem from many origins, including abnormal loading, inflexibility, hypermobility, neuromuscular weakness, altered kinesthesia, and/or structural malalignment. ${ }^{5}$ Given the diverse array of origins that can impact the athlete's ability to safely demonstrate linking in sport, it is important that clinicians consider and monitor various physical, physiological, and biopsychosocial qualities when determining where an athlete fits on the RTS continuum. For example, one inactive muscle, compromised inert tissue integrity, poor loading strategies, or exceeding tissue workload thresholds can lead to physical breakdown or poor performance by an athlete attempting to RTS. Research has shown how a loss of range of motion, strength or sequencing at one joint or segment can affect a joint or segment proximal or distal to the dysfunctional driver; this notion was coined in 2007 by Wainner et al. as Regional Interdependence. $^{6-17}$ It can be agreed that a multidisciplinary team is always beneficial for the athlete when trying to RTS; however, in today's professional sports atmosphere, different expectations and timelines may be put on the athlete by different individuals of the rehabilitation team. The referring surgeon may have a timeline based on biological healing, whereas the physical therapist may use more of a timeline based on functional returns, and the strength and conditioning coach's usage of strength and power as their determining factors for return. In an effort to systematically foster efficiency throughout the RTS process, we have laid out a comprehensive, progressive continuum of checks and balances referred to as the RTS Clearance Continuum (RTSCC).

The RTSCC is being proposed to the profession as a flexible framework aimed to foster multidisciplinary communication of checkpoints and workload expectations between stakeholders for team sport athletes as they progress through the RTS process (Fig 1).

\section{Phase I: Repair Phase}

The repair phase attempts to facilitate the normal healing process. An effort is made to minimize swelling, gain pain free range of motion, and ensure muscle activation of the affected site in both open and closed chain positions. Body positioning, modalities, and compression garments contribute to controlling the negative effects associated with this phase. It is of utmost importance to identify the underlying etiology of presented symptoms so that proper management can ensue. Too often, clinicians tend to simply test tissue integrity or unknowingly load the healing tissue too quickly. Understanding the biology of healing and the properties and qualities of the affected tissues will ensure sound clinical judgment.

\section{Phase II: Rehabilitation and Recovery Phase}

The first goal of this phase is to restore normal preinjury arthro-kinematics. It is important for clinicians to remember that compromises within inert tissue will require a more dynamic neuromuscular role for the contractile tissue, especially when stability issues are discovered. An athlete found with bony architecture and inert tissue deficiencies should experience a longer recovery period, which must be managed effectively if the athlete plans to RTS at a high-performance level. Once the athlete can habitually activate the appropriate 


\section{STAGED RTS CLUSTERED PERFORMANCE MODEL THE RETURN TO SPORT CLEARANCE CONTINUUM (RTSCC)}

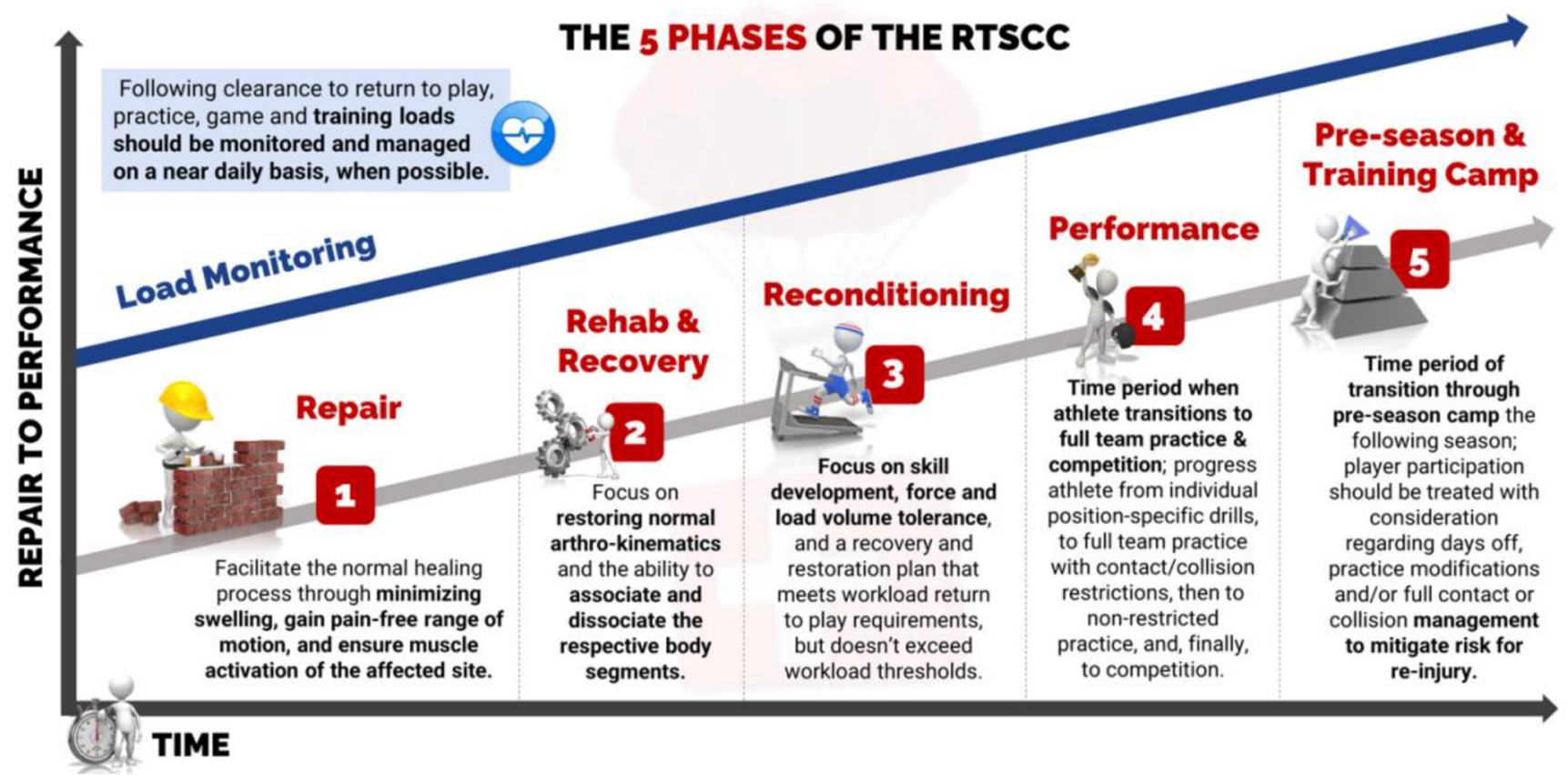

Fig 1. The 5 phases of the Return to Sport Clearance Continuum (RTSCC) start from the. repair phase where initial healing is occurring post surgically. This is where swelling will be minimized, range of motion will be increased, and proper muscle activation will occur. Next will be the rehabilitation and recovery phase, where normal arthrokinematics are going to be restored. Then, the athlete will move to the reconditioning phase, where the focus will be on skill and force development, along with load volume tolerance. The athlete will then progress to the performance phase, where the athlete will transition to full team practice and competition. Finally, they will progress to the preseason/training camp phase, where they will properly be managed for the upcoming season after injury. Throughout the continuum training loads need. to be monitored on a near daily basis when possible, to avoid overloading the healing tissues.

contractile tissue in a repetitive, noncompensating manner, tissue strength can be developed. It's important to recognize that high-level athletes are often excellent compensators. Due to their systemic involvement in sport, they develop chronic injuries and movement compensations to continue to produce the skills required for successful sport participation. Initially, strength development should address the local affected area, and, as strength builds, it will become necessary to maintain positions and postures while using developmental progressions of supine, prone, side-sit, quadruped, high kneel, half kneel, standing, squat, and lunge positions. Once postural stability is established, the next goal of this phase transitions toward ensuring that the athlete can demonstrate the ability to associate and dissociate inter-limb segments. We can exemplify this transition through an athlete who must return to throwing a baseball. When throwing, there is no initial turning of the trunk, hips, and pelvis, but this movement progresses to simultaneous turning, and finally to differentiated turning. This sequential progression of disconnecting and connecting of the segments leads to increased amounts of stored energy and conservation of momentum, commonly referred to as kinetic linking. ${ }^{17}$

\section{Phase III: Reconditioning Phase}

The reconditioning phase is without question the least understood and most presumptuous part of the recovery process. The reconditioning plan should address skill development, force and load volume tolerance, and include an RTS plan that systematically exposes the athlete to workloads replicating those of sport requirements without exceeding workload capacity. Seamlessly bridging and monitoring the gap between rehabilitation and performance is much easier said than done. As renowned reconditioning specialist Bill Knowles points out, reconditioning shifts the focus from the injury to the athlete. ${ }^{18}$ Load and force demands on the injured tissue are of primary concern during the reconditioning phase. The progression of these demands and adaptation of load tolerance should be based on the athletes competitive expectation levels. The biggest concerns for suboptimal RTS during this period are training load application oversights and failed load transfer. Abrupt, substantial tissue overload 
within a condensed time frame is a frequent training load application oversight. By developing a conservative and progressive plan, probability of such training load application oversights can be reduced. Studies have found a tendency for a $28 \%$ injury risk reduction for every additional month prior to return to training following anterior cruciate ligament reconstruction (ACLR) in professional soccer players. ${ }^{19}$ Although advancement to the performance phase may be prolonged, the additional time should be viewed as necessary for RTS success rather than an inconvenient delay. Failed load transfer is how effectively load can be managed throughout the segments within the system. Without effective inter-segmental force transfer, the athlete will be ill-prepared to succeed when performing sport-specific actions in chaotic and reactive environments. The use of accelerometers, heart rate and sleep monitors, force plates, and movement capturing devices can identify movement patterns, kinematics, and kinetics for helping to develop a customized plan. However, application of these data can only be as successful as the expertise and common sense of the care providers.

\section{Phase IV: Performance Phase}

The performance phase can be defined as the time period between the athlete's first return to practice or competition postinjury and subsequent return to practice or competition the following season. When initially reintegrating the athlete into sport-specific practice postinjury, it is recommended that the athlete is exposed to a proportion of position-specific drills repetitively performed at full speed while limiting physical interathlete contact. After successful initial reintegration without setback, the athlete should begin participating in full team practice with contact restrictions. Barring a setback, the athlete should ultimately return to full competition without any restrictions within the performance phase. This is where the journey usually ends regarding the comeback trail for most physicians and clinicians.

\section{Phase V: Training Camp/Preseason}

Training camp of the following season should be treated with consideration regarding days off and practice modifications. Just because an athlete had successful RTS within the first year postinjury does not infer that success will continue. For example, literature indicates that it may take up to 2 years to achieve baseline joint biological health and function following ACLR. ${ }^{20}$ The preseason strength and conditioning program should be designed with the intent of developing strength and fitness base levels necessary for meeting both the physical and mental demands of the chosen sport. There are other factors that must be considered when considering participation practice and competition activities the following pre-season. Talent levels of replacement players, time of season, and contract status are just a few of the many variables which may influence an athlete's RTS. Although the athlete may not exhibit mental and physical preparedness to participate, these factors weigh into the holistic decision as to whether or not it is in the best interest of the athlete to RTS.

\section{Load Monitoring}

Tremendous attention has been paid to "load" management and recovery in professional team sport settings over the past 10 years. These may be the most neglected parts of the RTS process by clinicians. "Load" is a derivative of many forms, including heart rate, global positioning system, inertial measurement units, perceived effort, and duration. Recovery modalities range from simple things like contrast tubs to float tanks and cryo-chambers. Sleep, perceived well-being, and heart rate variability data interpretation have also been used to better understand recovery in athletes. Load and recovery data collection and interpretation has led to entire sports science departments being phased into the sports medicine department for professional teams. The integration of technology into RTS progression has become a staple in high-performance environments. Regardless of performance environment and available technology, it is recognized that the balance between stress (e.g., load) and recovery determines rate of adaptation, and RTS progression. The real value of technology integration has little to do with the technology itself. Rather, the "art" of sports science, or how the technology is applied, is what dictates RTS success. Understanding the data, determining which metrics are important and how they can be used to make informed decisions, and finding simple, noninvasive ways to systematically collect and monitor information are what allows for the RTS process to include relevant actionable conclusions that are useful to athletes, coaches, and managers.

This will be a maintenance stage of the RTSCC. Failure to do so could cause the player to shift in and out of different parts of the continuum.

The following sections will break down lowerextremity (LE) injuries in particular, and how the suggested RTSCC can help shed some light in a complete return to play process

\section{Lower Extremity Focus}

The question "When can I return to sport?" is the first to be asked after an athlete suffers an injury. At present, we have a limited understanding of the RTS decisionmaking after LE injury. Despite technological advancements, time continues to be the focus of RTS decision-making. A recent systematic review identified time from injury as the sole criteria for RTS in nearly 
$50 \%$ of qualifying studies. ${ }^{2}$ Some studies have exemplified the fact that RTS should be delayed anywhere from 9 months to 2 years, citing a reduction of reinjury rates up to $51 \%$ for each month RTS was delayed. ${ }^{21,22}$ While biological healing is an important factor in RTS, a paradigm shift has focused on a criterion-based approach to RTS. Reported objective criteria include strength testing, hop testing, balance/postural control, qualitative movement assessment, cognitive decision based movement, and patient-reported outcome measures. More recently, quantitative measures including 3-dimensional biomechanics have been discussed in the literature to help with the RTS process. ${ }^{23}$ However, criterion-based approaches are poorly enforced, as only $13 \%$ of studies report objective measurements as a criteria for RTS. ${ }^{24}$ The fact remains that there are studies emerging that have shown significant reduction in reinjury rates in individuals who passed objective criteria before RTS. ${ }^{21,25}$ The current state of RTS testing is underwhelming, as it does not maximize the use of technology nor fully appreciate context specificity of RTS progression. In the following section, we review the current state of RTS testing.

\section{Strength Testing}

Isolated strength deficits often persist long after initial injury. Poor quadricep strength has been associated with poor performance at 9 months post surgically, and sometimes up to $>50 \%$ failed to have limb symmetry index (LSI) $>90 \%$ at 9 months. ${ }^{21,26}$ Traditional strength testing for RTS has been performed using isokinetic dynamometry. Agonist:antagonist strength ratios and LSI are the 2 primary strength testing protocols used in RTS criteria. For example, Tyler et al. ${ }^{27}$ demonstrated that professional hockey players with an adductor:abductor strength ratio of $<.80$ were at a $17 \times$ increased risk for sustaining an adductor strain over the course of a season. Limb symmetry has been criticized with strength testing as bilateral strength deficits tend to exist after LE injury. ${ }^{28}$ More recently specialized devices with force transducers and handheld dynamometers have been used to gather baseline measures and normative data at the beginning of the season as a base of comparison. Baseline and serial strength testing provide objective data for regular monitoring and individualized RTS metrics. Baseline testing is advantageous in the professional athlete population by providing a pre-injury comparison for potential postinjury data.

\section{Hop Testing}

Functional hop testing is frequently used in RTS testing. Single-leg hop for distance, triple hop for distance, crossover hop for distance, and the 6-m timed hop are common tests used and reported in the literature. It is commonly accepted that a LSI of $>85 \%$ of the injured side is appropriate to determine readiness to RTS. ${ }^{29}$ However, LSIs have been an area of scrutiny in recent years, and for good reason. Asymmetries are task- and metric-specific, and thus, a global percentage threshold of clinical relevance should be avoided. ${ }^{30}$ LSIs have been shown to overestimate knee function in comparison with preoperative hop testing. ${ }^{31}$ In a recent meta-analysis, Kotsifaki et al. $^{32}$ identified that kinematic and kinetic deficits in athletes were detected despite adequate single-leg hop for distance performance. Furthermore, there was high agreement that a single-leg countermovement jump may be more sensitive in identifying deficits in individuals post-ACLR. ${ }^{29}$ It is important that sports medicine professionals consider the principle of training specificity in RTS; adaptations to vertically or horizontally orientated training promote vector-specific adaptations. ${ }^{33}$ Continuing to train and test the horizontal jump, and its mechanics is widely useful especially when thinking of training speed and deceleration, but the unilateral and bilateral countermovement jump should be considered as components of LE RTS testing to identify deficits in vertical force propulsion and absorption. ${ }^{32,34}$

\section{Postural Control/Dynamic Balance}

Measures of balance and postural control as RTS criteria have been discussed in the literature and should be considered as a component of testing. Research by Plisky et al. ${ }^{35}$ demonstrated, athletes with an anterior right/left reach distance difference of greater than $4 \mathrm{~cm}$ on the Y-balance test were 2.5 times more likely to sustain a LE injury. Furthermore, females with a composite reach distance less than $94 \%$ of their limb length had a 6.5 times greater risk for injury. ${ }^{35}$

\section{Qualitative Movement}

Alterations in movement strategies tend to persist in LE injury, but it is unclear whether altered movement strategies are a sequela of injury, or if they existed before injury. In a 3-dimensional biomechanics study by Boo et al., ${ }^{36}$ individuals who underwent ACLR demonstrated reduced energy absorption contribution from an ankle strategy when landing from a drop vertical jump. This altered movement strategy can be associated with decreased triple flexion of the LE and poor deceleration qualities. Use of established functional assessment tools including the drop vertical jump, and landing error scoring system can be used to identify faulty movement patterns and differentiate individuals who have sustained LE injuries. Recent work by King et al. ${ }^{23}$ demonstrated reductions in ipsilateral trunk side flexion and increased pelvic rotation in the direction of travel associated with improved cutting performance in a cohort of 205 patients with athletic groin pain. 3D analysis of trunk and LE positioning can be effective in identifying risk factors in 


\section{STAGED RTS CLUSTERED PERFORMANCE MODEL RTSCC TESTING PROGRESSION WITH SAMPLE TESTS}

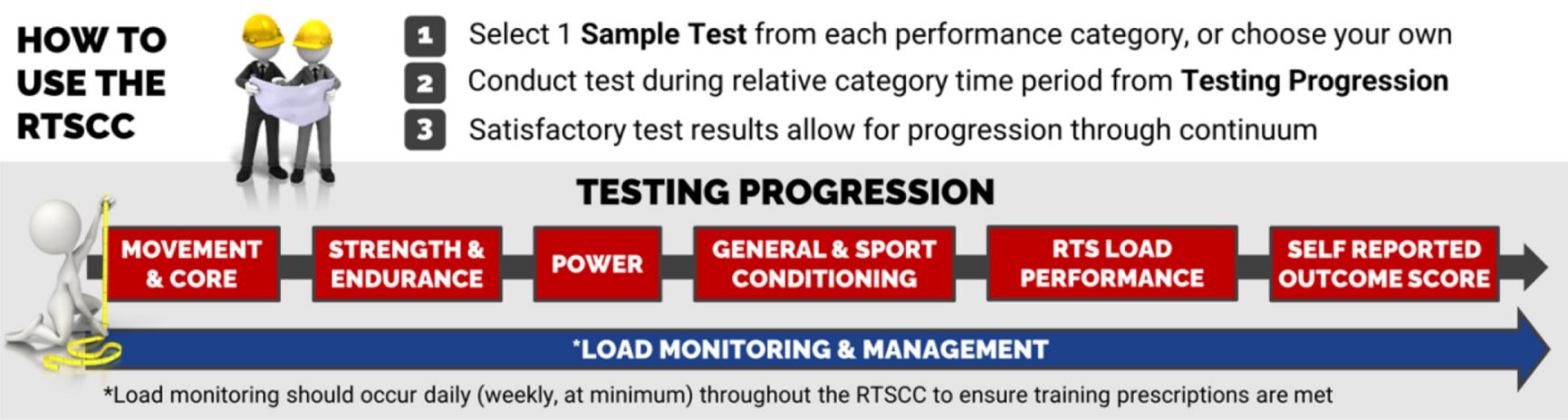

SAMPLE TESTS, BY PERFORMANCE CATEGORY

\begin{tabular}{|l|}
\hline Movement \& Core \\
- Motion Capture \\
- FMS/SFMA/QMA \\
- SEBT/Y-Balance \\
- McGill \\
- Bunkey \\
- Watkins \\
\hline
\end{tabular}

\begin{tabular}{|l|}
\hline \multicolumn{1}{|c|}{ Strength \& } \\
Endurance \\
- Dynamometer \\
- Force Plate \\
- Isokinetic \\
- 1RM (or \%1RM) \\
\hline
\end{tabular}
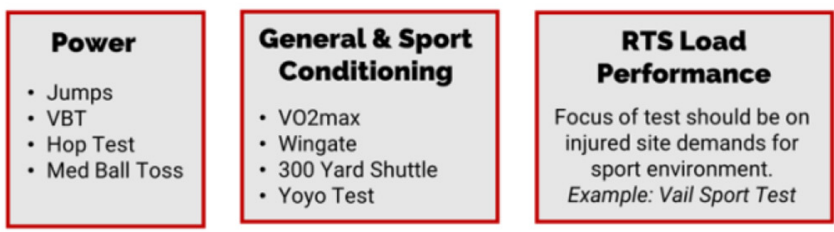

Load Monitoring: Accelerometry, GPS, Heart Rate, RPE, Subjective Wellness Questionnaires, Psychological Readiness

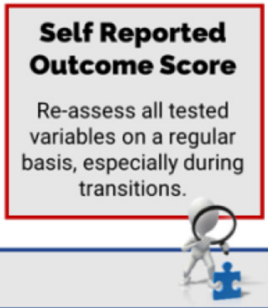

Fig 2. Return to Sport Clearance Continuum (RTSCC) Sample testing: Throughout the continuum proper testing needs to occur to make sure that the athlete is ready to progress onto the next stage. A measure of progression should be tested in the following categories; movement and core, strength and endurance, power, general and sport conditioning, load performance testing, and a self-reported outcome score. Satisfactory results from testing result in the athlete moving on in the continuum. Load monitoring should continue to happen throughout using methods like accelerometry, global positioning system (GPS), heart rate, rate of perceived exertion (RPE), subjective wellness questionnaires, and psychological readiness. Continuous monitoring ensures that the athlete is at the right phase of the continuum and adjustments can be made if need be. (1RM, one rep maximum; FMS, Functional Movement Screen; QMA, quality of movement assessment; SEBT, Star Excursion Balance Test; SFMA, Selective Functional Movement Assessment; VBT, Velocity-based training.)

movement quality as part of a comprehensive RTS testing battery.

\section{Advancements in Testing/Special Considerations}

Significant strides have been made in improving outcomes with RTS after LE injury. Two decades ago, ACL tears were seen as catastrophic injuries to field/ court athletes. Recent prospective cohort studies have demonstrated RTS rates of $>85 \%$ in individuals who underwent primary ACLR. ${ }^{21}$ In nearly all individuals who suffer soft-tissue injuries including hamstring and groin injuries RTS, however, recurrence rates can be high and result in greater time loss from sport. While returning to competitive sport after LE injury occurs in many athletes, the alarming fact is the relatively low number of athletes who RTS at their preinjury level of competition. Some studies report only 55\% returned to sport at the previous level of function. ${ }^{37}$ While many athletes RTS after LE injury, remaining in sport at a high level presents some clinical challenges. A thorough
RTSCC with criterion-based decision making should be used to promote sustainability in RTS.

The need for comprehensive objective RTS criteria including both quantitative and qualitative measures cannot be overstated for continuous monitoring throughout the duration of the RTSCC. Time and biological healing should be considered but cannot be used in isolation. It is the opinion of the authors that RTS testing be specific to the movement capacity, strength/ power, and energy system demands of the athlete's sport. The extent to which movement analysis can be performed will vary based on institutional resource availability. As stated earlier, it is not uncommon to have 3-dimensional motion analysis, force plates, and training load monitoring capabilities available to the training staff in elite sport environments. These resources must be continually used throughout the process to assess readiness in the continuum and to see if changes in the program need to be made.

Advancements in LE strength and power testing have paralleled technological developments in sports science. Fixed dynamometer testing is a reliable and valid 


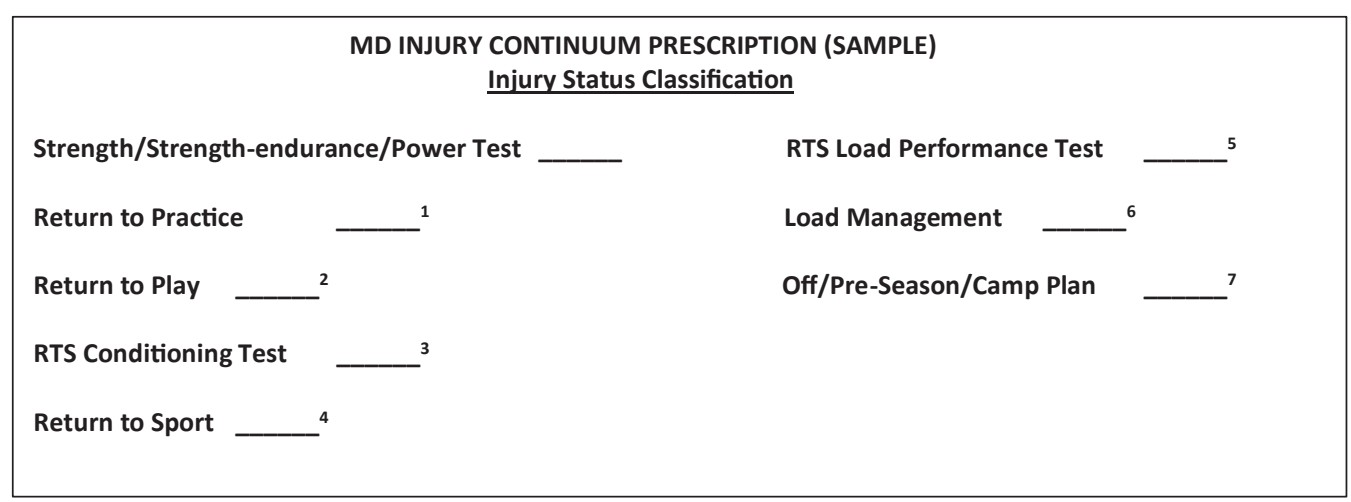

Fig 3. A sample MD prescription that would be provided to the athlete/team, noting what stage the athlete is in the continuum. The athlete moves through the continuum through proper communication between the rehabilitation team and the treating $\mathrm{MD} /$ surgeon. Proper testing and completion of criteria will determine what level the athlete is at in the continuum. The definitions for each phase are explained to follow: ${ }^{1}$ Return to Practice is the integration of player back to individual skills portion of practice. ${ }^{2}$ Return to Play is the integration of the player back into team activities with contact and collision restrictions. ${ }^{3} \mathrm{RTS}$ Conditioning Testing general conditioning test to identify the base, sport-specific conditioning test to identify the energy system used in the specific sport. ${ }^{4}$ Return to Sport is full participation without restriction starting with practice before a live game. ${ }^{5}$ RTS Load Performance Test - challenge the injured area with a dynamic sport-specific muscular endurance test battery to ensure ability for the injured segment to meet the load demands of the sport. ${ }^{6}$ Load Management is the monitoring, modifying, and management of practice, game, and training forces for the remainder of the season. ${ }^{7}$ Pre-Season Plan should focus on developing base strength that can help to carry them through the season. If they have had 1 or 2 consecutive off-season surgeries, they will be starting the next off season at a base strength deficit. The preseason planning must look at how to protect them from being overloaded due to the rigors of preseason camp. Summer Sports camps should also be placed in this category.

technique for maximal isometric and eccentric strength. ${ }^{1,38}$ In addition, the clinical use of dual-force plate technologies has allowed for evaluation of force-time characteristics for various upper and lower body movements. The isometric mid-thigh pull is a test designed to quantify maximal isometric strength and rate of force development and has been demonstrated to be a valid way to determine asymmetrical strength in athletes. ${ }^{39,40}$ To date, there are no publications on use of the isometric mid-thigh pull as RTS criteria, but this may be helpful in determining LE strength and rate of force development ( in injured populations, but more research is needed at this time. ${ }^{41}$ Jump testing with the addition of force plate technologies allow the rehabilitation staff to look beyond outputs such as vertical jump height when assessing the athlete. Force/time and jump phase specific characteristics can provide insight into specific neuromuscular qualities that may be valuable in assessing the rehabilitated athlete. Calculated metrics such as the dynamic strength index can be used to reflect the extent to which an athlete is able to apply force dynamically in relation to their maximal force capabilities. The primary goal of force-velocity profiling with the rehabilitating athlete would be to prescribe targeted interventions to shift the force velocity curve to the right, thus maximizing strength and power qualities. With the increasing accessibility of force plate technologies in the clinical setting, neuromuscular assessments of maximal strength and various jump qualities should be considered as a component of a RTSCC.
In physical therapy, it is not uncommon to use clinical prediction rules during evaluation to identify pathology or to select an appropriate treatment plan based on symptoms. Since it is very difficult to use a single test to identify a specific pathology, it has become more common to cluster groups of tests together. Current RTS efforts have previously attempted to draw conclusions from a single test or a group of similar tests to assist in making the most informed decision. During the RTSCC, we are advocating for a battery of test options and categories to be assessed and performed at different periods throughout the recovery process, as outlined to follow (Fig 2).

\section{Food for Thought}

The first part of any continuum is to determine the status of the clinical examination. The injury must then go through a normal physiological repair and regeneration process. A rehabilitation and recovery plan is then executed until certain clinical milestones are achieved. The focus is then switched to the athlete and away from the injury until they are physiologically and psychologically deemed ready to return to individual and team activities. It is following this stage that an appropriate RTS testing protocol should be implemented to identify if the athlete possesses the workload capacity for returning to unrestricted sport competition. Upon clearance, it will be important to record forces and revise workloads. In our opinion, the last part of the continuum is to design and implement a 
comprehensive preseason plan that monitors loads, repetitions, recovery models, and including days off when possible. It is imperative to note that although all athletes will eventually go through the continuum in varied amounts of time, the progression through this continuum is criteria based. Athletes need to meet the criteria for progressing through the phases so that safe RTS and optimal return to performance is ultimately achieved. Some may achieve this in a timeline that goes parallel with physiologic healing while others may lag behind due to the fact that criteria are not met to move forward (Fig 3).

The inclusion of all the stakeholders (player, coach, physician, athletic trainer, physical therapist, strength coach, nutritionist, and in some instances a sport psychologist) who may be able to provide feedback or insight regarding any of the phases or the athlete's physiological or psychological well-being is essential.

\section{References}

1. Ryan S, Kempton T, Pacecca E, Coutts AJ. Measurement properties of an adductor strength assessment system in professional Australian footballers. Int J Sports Physiol Perform 2019;14:256-259.

2. Burgi CR, Peters S, Ardern CL, et al. Which criteria are used to clear patients to return to sport after primary ACL reconstruction? A scoping review. Br J Sports Med 2019;53: 1154-1161.

3. Taberner M, Allen T, Cohen DD. Progressing rehabilitation after injury: Consider the 'control-chaos continuum. Br J Sports Med 2019;54:116-117.

4. Kibler B. Pathophysiology of throwing injuries: The kinetic chain. In: Dines J, Altchek D, Andrews JR, eds. Sports medicine of baseball. Philadelphia, PA: Wolter Kluwer Health, 2012;30-36.

5. Deppen R. From floor to the core. In: Donatelli RA, ed. Sport-specific rehabilitation. Philadelphia, PA: Elsevier, 2006.

6. Robb AJ, Fleisig G, Wilk K. Passive ranges of motion of the hips and their relationship with pitching biomechanics and ball velocity in professional baseball players. Am J Sports Med 2010;38:2487-2493.

7. Ellera-Gomes JL, de Castro JV, Becker R. Decreased hip range of motion and non-contact injuries of the anterior cruciate ligament. Arthroscopy 2008;24:1034-1037.

8. Bedi A, Warren RF, Oh YK, Wojtys EM, Oltean HN, Kelly BT. Restriction of hip internal rotation is associated with an increased risk of ACL injuries in NFL combine athletes: A clinical and biomechanical study. Orthop J Sports Med 2013;1 (4 suppl).

9. Vad VB, Bhat AL, Basari D. Low back pain in professional golfers. Am J Sports Med 2004;32:494-497.

10. Larson CM, Pierce BR, Giveans MR. Treatment of athletes with symptomatic intra-articular hip pathology and athletic pubalgia/sports hernia: A case series. Arthroscopy $2011 ; 27: 768-775$.

11. Lewis CL, Ferris DP. Walking with increased ankle pushoff decreases hip muscle moments. J Biomech 2008;41:2082-2089.
12. Bullock-Saxton JE, Janda V, Bullock MI. The influence of ankle sprain injury on muscle activation during hip extension. Int J Sports Med 1994;15:330-334.

13. Hodges PW, Richardson CA. Contraction of the abdominal muscles associated with movement of the lower limb. Phys Ther 1997;77:132-142.

14. Stodden DF, Fleisig G, McLean SP, Kyman SL. Relationship of pelvis and upper torso kinematics to pitched baseball velocity. J Appl Biomech 2001;17:164-172.

15. Panjabi MM. The stabilizing system of the spine. Part I. Function, dysfunction, adaptation, and enhancement. J Orthop Sports Phys Ther 2007;37:658-660.

16. Erhard R, Bowling R. The recognition and management of the pelvic component in low back and sciatic pain. Bull Orthop Sect Am Phys Ther Assoc 1977;2:4-15.

17. Wainner RS, Whitmann JM, Cleland JA, Flynn TW. Regional interdependence: A musculoskeletal examination model whose time has come. J Orthop Sports Phys Ther 2007;37:658-660.

18. Knowles B. Reconditioning: A performance based response to injury. In: Joyce D, Lewindon D, eds. Sports injury prevention and rehabilitation. London: Routledge, 2016.

19. Della Villa F, Hägglund M, Della Villa S, et al. High rate of second ACL injury following ACL reconstruction in male professional footballers: An updated longitudinal analysis from 118 players in the UEFA Elite Club Injury Study. Br J Sports Med 2021;55:1350-1356.

20. Nagelli CV, Hewett TE. Should return to sport be delayed until 2 years after anterior cruciate ligament reconstruction? Biological and functional considerations. Sports Med 2017;47:221-232.

21. Grindem H, Snyder-Mackler L, Moksnes H, Engebretsen L, Risberg MA. Simple decision rules can reduce reinjury risk by $84 \%$ after ACL reconstruction: The Delaware-Oslo ACL cohort study. Br J Sports Med 2016;50: 804-808.

22. Kemp JL, Risberg MA, Schache AG, Makdissi M, Pritchard MG, Crossley KM. Patients with chondrolabral pathology have bilateral functional impairments 12 to 24 months after unilateral hip arthroscopy: A cross-sectional study. J Orthop Sports Phys Ther 2016:46:947-956.

23. King E, Franklyn-Miller A, Richter C, et al. Clinical and biomechanical outcomes of rehabilitation targeting intersegmental control in athletic groin pain: Prospective cohort of 205 patients. Br J Sports Med 2018;52: 1054-1062.

24. Barber-Westin SD, Noyes FR. Factors used to determine return to unrestricted sports activities after anterior cruciate ligament reconstruction. Arthroscopy 2011;27: 1697-1705.

25. Kyritsis P, Bahr R, Landreau P, Miladi R, Witvrouw E. Likelihood of ACL graft rupture: Not meeting six clinical discharge criteria before return to sport is associated with a four times greater risk of rupture. Br J Sports Med 2016;50:946-951.

26. Waldén M, Hägglund M, Magnusson H, Ekstrand J. ACL injuries in men's professional football: a 15-year prospective study on time trends and return-to-play rates reveals only $65 \%$ of players still play at the top level 3yearsafter ACL rupture. Br J Sports Med 2016;50: 744-750. 
27. Tyler TF, Nicholas SJ, Campbell RJ, McHugh MP. The association of hip strength and flexibility with the incidence of adductor muscle strains in professional ice hockey players. Am J Sports Med 2001;29:124-128.

28. Hiemstra LA, Webber S, Macdonald PB, Kriellaars DJ. Contralateral limb strength deficits after anterior cruciate ligament reconstruction using a hamstring tendon graft. Clin Biomech 2007;22:543-550.

29. Noyes FR, Barber SD, Mangine RE. Abnormal lower limb symmetry determined by function hop tests after anterior cruciate ligament rupture. Am J Sports Med 1991;19:513-518.

30. Bishop C. Interlimb asymmetries: Are thresholds a usable concept? Strength Cond J 2021;43:32-36.

31. Wellsandt E, Failla MJ, Snyder-Mackler L. Limb symmetry indexes can overestimate knee function after anterior cruciate ligament injury. J Orthop Sports Phys Ther 2017;47: 334-338.

32. Kotsifaki A, Korakakis V, Whiteley R, Van Rossom S, Jonkers I. Measuring only hop distance during single leg hop testing is insufficient to detect deficits in knee function after ACL reconstruction: A systematic review and meta-analysis. Br J Sports Med 2019;54:139-153.

33. Moran J, Ramirez-Campillo R, Liew B, et al. Effects of vertically and horizontally orientated plyometric training on physical performance: A meta-analytical comparison. Sports Med 2021;51:65-79.

34. Kotsifaki A, Korakakis V, Graham-Smith P, Sideris V, Whiteley R. Vertical and horizontal hop performance: Contributions of the hip, knee, and ankle. Sports Health 2021;13:128-135.
35. Plisky PJ, Rauh MJ, Kaminski TW, Underwood FB. Star excursion balance test as a predictor of lower extremity injury in high school basketball players. J Orthop Sports Phys Ther 2006;36:911-919.

36. Boo ME, Garrison JC, Hannon JP, et al. Energy absorption contribution and strength in female athletes at return to sport after anterior cruciate ligament reconstruction: Comparison with healthy controls. Orthop J Sports Med 2018;6:232596711875952.

37. Ardern CL, Taylor NF, Feller JA, Whitehead TS, Webster KE. Sports participation 2 years after anterior cruciate ligament reconstruction in athletes who had not returned to sport at 1 year. Am J Sports Med 2015;43: 848-856.

38. Opar DA, Williams MD, Timmins RG, Hickey J, Duhig SJ, Shield AJ. Eccentric hamstring strength and hamstring injury risk in Australian footballers. Med Sci Sports Exerc 2015;47:857-865.

39. Thomas C, Dos'Santos T, Comfort P, Jones P. Betweensession reliability of 519 common strength- and powerrelated measures in adolescent athletes. Sports (Basel) 2017;5:15.

40. Dos'Santos T, Thomas C, Jones PA, Comfort P. Assessing muscle strength 394 asymmetry via a unilateral stance isometric mid-thigh pull. Int J Sports Physiol Perform 2017;12:505-511.

41. Jordan MJ, Aagaard P, Herzog W. Lower limb asymmetry in mechanical muscle function: A comparison between ski racers with and without ACL reconstruction. Scand J Med Sci Sports 2014;25(3). 